\title{
Ballistic rectification of vortex domain wall chirality at nanowire corners
}

\author{
K. Omari, ${ }^{1}$ R. C. Bradley, ${ }^{1}$ T. J. Broomhall, ${ }^{1}$ M. P. P. Hodges,${ }^{1}$ M. C. Rosamond, ${ }^{2}$ \\ E. H. Linfield, ${ }^{2}$ M.-Y. Im, ${ }^{3,4}$ P. Fischer, ${ }^{5,6}$ and T. J. Hayward ${ }^{1}$ \\ ${ }_{1}^{1}$ Department of Materials Science and Engineering, University of Sheffield, Sheffield S1 3JD, United Kingdom \\ ${ }^{2}$ School of Electronic and Electrical Engineering, University of Leeds, Leeds LS2 9JT, United Kingdom \\ ${ }^{3}$ Center for X-Ray Optics, Lawrence Berkeley National Laboratory, Berkeley, California 94720, USA \\ ${ }^{4}$ Daegu Gyeongbuk Institute of Science and Technology, Daegu 711-873, South Korea \\ ${ }^{5}$ Materials Sciences Division, Lawrence Berkley National Laboratory, Berkeley, California 94720, USA \\ ${ }^{6}$ Department of Physics, University of California, Santa Cruz, California 94056, USA
}

(Received 3 September 2015; accepted 13 November 2015; published online 2 December 2015)

\begin{abstract}
The interactions of vortex domain walls with corners in planar magnetic nanowires are probed using magnetic soft X-ray transmission microscopy. We show that when the domain walls are propagated into sharp corners using applied magnetic fields above a critical value, their chiralities are rectified to either clockwise or anticlockwise circulation depending on whether the corners turn left or right. Single-shot focused magneto-optic Kerr effect measurements are then used to demonstrate how, when combined with modes of domain propagation that conserve vortex chirality, this allows us to dramatically reduce the stochasticity of domain pinning at artificial defect sites. Our results provide a tool for controlling domain wall chirality and pinning behavior both in further experimental studies and in future domain wall-based memory, logic and sensor technologies. (C) 2015 AIP Publishing LLC. [http://dx.doi.org/10.1063/1.4936565]
\end{abstract}

Devices based on the motion of domain walls (DWs) in magnetic nanowires have been in development for over a decade. ${ }^{1-3}$ While simple descriptions visualize DWs as rigid particles, DWs actually have complex internal magnetization structures $^{4}$ that change dynamically as they propagate. ${ }^{5-7}$ Key to understanding DW structure is the concept of chirality, which describes the sense of magnetization rotation across the DW. For example, in the case of vortex DWs (VDWs), chirality dictates whether their internal magnetization rotates clockwise $(\mathrm{CW})$ or anticlockwise $(\mathrm{ACW})$ around a central out-of-plane core.

Chirality has a strong influence on DW behavior: VDWs with opposite chiralities pin differently at notches, resulting in stochastic depinning field distributions in systems where chirality is ill-defined. ${ }^{8,9}$ Furthermore, chirality dictates the paths of DWs in branched nanowires, and therefore the geometry of Dirac strings in artificial spin-ice lattices. ${ }^{10}$ In systems where DW structure can be stabilized, chirality also offers a binary degree of freedom, leading to proposals for chirality-based logic networks. ${ }^{11}$

DWs injected using injection pads or current lines have random chiralities, leading to uncontrolled pinning behaviors. However, by breaking the symmetry of these features, chirality can be controlled at the point of injection. ${ }^{10,12}$ Controlling and manipulating chiralities during propagation is more challenging, but can be achieved by interacting DWs with orthogonal nanowire sections, ${ }^{13}$ large notches, ${ }^{14}$ or enddomains; ${ }^{15}$ however, these approaches all involve the introduction of large defects into the nanowires. Pulsed rotating fields can be used to select DW chirality in ring-shaped nanowires, ${ }^{16}$ but this approach would be complex to implement in devices.

In this paper, we propose a simple method of controlling DW chirality in continuous nanowires. We show that DWs propagated ballistically into sharp nanowire corners are reliably rectified to either $\mathrm{CW}$ or $\mathrm{ACW}$ chirality depending on whether the corner turns left or right. We then demonstrate how this approach can be exploited to dramatically reduce the stochasticity of pinning at artificial defect sites.

To probe the interaction of VDWs with corners we fabricated $400 \mathrm{~nm}$ wide nanowires, with geometries similar to that shown in Fig. 1(a), on $\mathrm{Si}_{3} \mathrm{~N}_{4}$ membranes using electron beam lithography with lift-off processing. Metallization with $40 \mathrm{~nm}$ of $\mathrm{Ni}_{80} \mathrm{Fe}_{20}$ was achieved by thermal evaporation. DWs were injected from the pads at the nanowires' left ends and propagated into corners with radii, $\mathrm{r}=1 \mu \mathrm{m}, 2 \mu \mathrm{m}$, or $5 \mu \mathrm{m}$ using applied magnetic fields. Nanowires with corners that turned both left (UP geometry) and right (DOWN geometry) were investigated. In some nanowires, a symmetrical double-notch with gap-size "g" was positioned before the corner to allow the initial chiralities of the DWs to be probed. To measure the VDWs' chiralities, we performed magnetic transmission soft X-ray microscopy (MTXM) experiments using beamline 6.1.2 at the Advanced Light Source, which provided spatial resolution $<25 \mathrm{~nm}$.

We performed initial measurements on both UP and DOWN geometry nanowires with $\mathrm{r}=1 \mu \mathrm{m}$ and $\mathrm{g}=160 \mathrm{~nm}$. The nanowires were first saturated into continuous configurations before $\mathrm{H}_{\mathrm{x}}=150-180 \mathrm{Oe}$ was applied to inject headto-head $(\mathrm{H} 2 \mathrm{H})$ VDWs from the pads and move them to the notches. The applied field was then removed to relax the VDWs and allow their chiralities to be determined. We then applied $\mathrm{H}_{\mathrm{x}}=300-350 \mathrm{Oe}$ to depin the VDWs from the notches and propagate them into the vertical sections of the nanowires beyond the corners, before the field was removed again to determine the VDWs' final chiralities.

Figs. 1(b)-1(d) present the results of these measurements. For the UP nanowires, VDWs with CW chirality were always observed after the corner, no matter whether the injected DWs had CW (Fig. 1(b)) or ACW (Fig. 1(c)) 
(a)

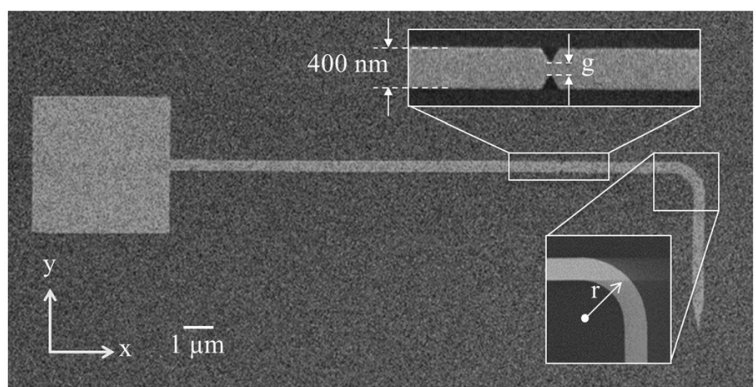

(b)
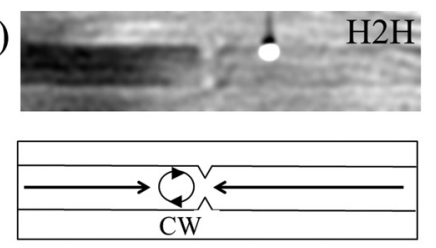

(c)
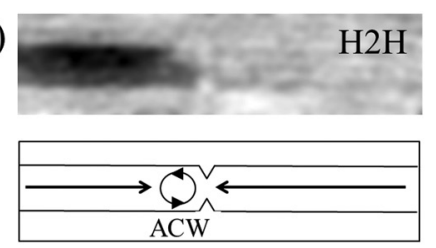

(d)
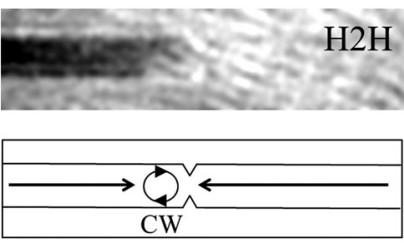

(e)
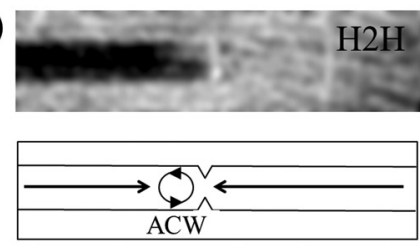

(f)

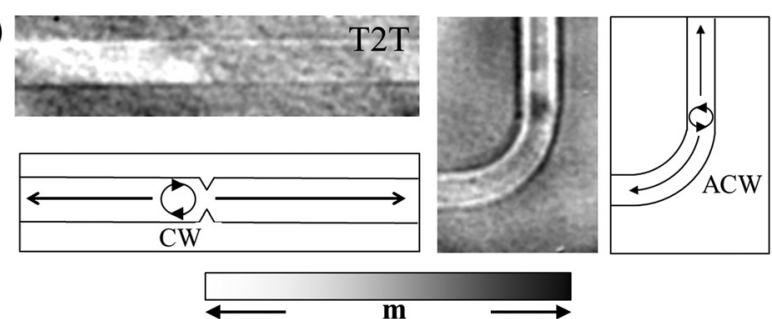

FIG. 1. (a) Scanning electron microscope image of a "DOWN" geometry nanowire. Enlargements of the notch and corner regions at which MTXM images were taken are shown inset. (b)-(f) MTXM images showing domain wall configurations before and after passing through the corners: (b) UP geometry nanowire with $\mathrm{CW}$ H2H VDW input. (c) UP geometry nanowire with ACW H2H VDW input. (d) DOWN geometry nanowire with CW H2H VDW input. (e) DOWN geometry nanowire with ACW H2H VDW input. (f) UP geometry nanowire with CW T2T VDW input.

chirality. Conversely, for the DOWN nanowires, only DWs with ACW chirality were observed after the corner (Figs. 1(d) and 1(e)). Together, these results indicated that the corners had a strong rectifying effect on the chirality of the DWs. The above trends were observed consistently over a total of 28 imaging cycles of the two nanowire geometries (for full details, see Ref. 17), allowing us to reject the hypothesis that the output chirality was random at a significant level of 5\%. Furthermore, the DWs' chirality flipped in nine events allowing us to reject the hypothesis that the rectification probability was less than $70 \%$.

To gain a deeper understanding of the rectification process, we performed micromagnetic simulations of ACW and CW DWs passing through $\mathrm{r}=1 \mu \mathrm{m}$ corners in UP geometry nanowires using the OOMMF software package. ${ }^{18}$ Simulations were performed on a $4 \mathrm{~nm} \times 4 \mathrm{~nm} \times 40 \mathrm{~nm}$ mesh using standard values for the material properties of $\mathrm{Ni}_{80} \mathrm{Fe}_{20}$ : saturation magnetization, $\mathrm{M}_{\mathrm{s}}=860 \mathrm{kA} / \mathrm{m}$, exchange stiffness, $\mathrm{A}_{\mathrm{ex}}=13 \mathrm{pJ} / \mathrm{m}$, magnetocrystalline anisotropy constant, $\mathrm{K}_{1}=0$ and damping constant, and $\alpha=0.02$. While approximating the nanowire as a $2 \mathrm{D}$ mesh was expected to make minor modifications to the details of the DW dynamics, this approach reduced computation time, while still allowing the basic rectification process to be understood. Initial H2H VDW structures were propagated into the corners by applying $+\mathrm{H}_{\mathrm{x}}$. Based on the experimental results, it was expected that ACW VDWs would be rectified to CW chirality, while CW VDWs would pass through the corner unchanged.

We initially performed simulations with a low propagation field of $\mathrm{H}_{\mathrm{x}}=50$ Oe to avoid turbulent DW dynamics. ${ }^{7}$ This was above the Walker breakdown (WB) field, ${ }^{5}$ but within the oscillatory regime of DW motion ${ }^{6}$ where dynamics were periodic, and, as we will show later, conserve vortex chirality in this nanowire geometry. Figs. 2(a) and 2(b) compare the simulated dynamics of DWs with $\mathrm{CW}$ and $\mathrm{ACW}$ chiralities as they were propagated into the corner. The $\mathrm{CW}$ VDW passed through without changing its chirality, while the ACW VDW was rectified to CW chirality, in good agreement with the MTXM results.

Detailed examination of the simulations revealed the cause of the rectifying effect: In the case of the CW VDW, the magnetization in the leading edge of the VDW pointed along $-\mathrm{y}$, parallel to that of the vertical section of the nanowire beyond the corner, allowing it to pass through easily. Conversely, for the ACW VDW, the magnetization in the leading edge was along $+\mathrm{y}$, antiparallel to the magnetization in the vertical section. As the DW approached the corner this produced a frustrated and unstable region of magnetization in front of it, allowing the nucleation of a $\mathrm{CW}$ vortex core at $10.2 \mathrm{~ns}$ (indicated by a blue arrow in the inset of Fig. 2(a)). As the DW moved further around the corner, the CW vortex core moved towards the center of the nanowire, while the original ACW core annihilated at the nanowire's upper edge, completing the rectification process.

Figures 2(c) and 2(d) present equivalent simulations for $\mathrm{H}_{\mathrm{x}}=250$ Oe, a field closer to that required to depin the DWs in the experimental measurements. Here, the dynamics were turbulent, with the DWs becoming extended and containing large numbers of vortex/antivortex cores at any given instant. Nevertheless, the rectification process still occurred, with both DWs resolving into CW VDW structure as they passed into the vertical section of nanowire. Clearly, the magnetization in the vertical section was able to bias the final nucleation events towards CW chirality as the DWs resolved into stable VDW configurations.

The mechanism we propose above explains why moving from the UP to the DOWN geometry nanowires caused rectification to ACW rather than $\mathrm{CW}$ chirality (Figs. 1(d) and 1(e)): in the DOWN geometry, the vertical section of 


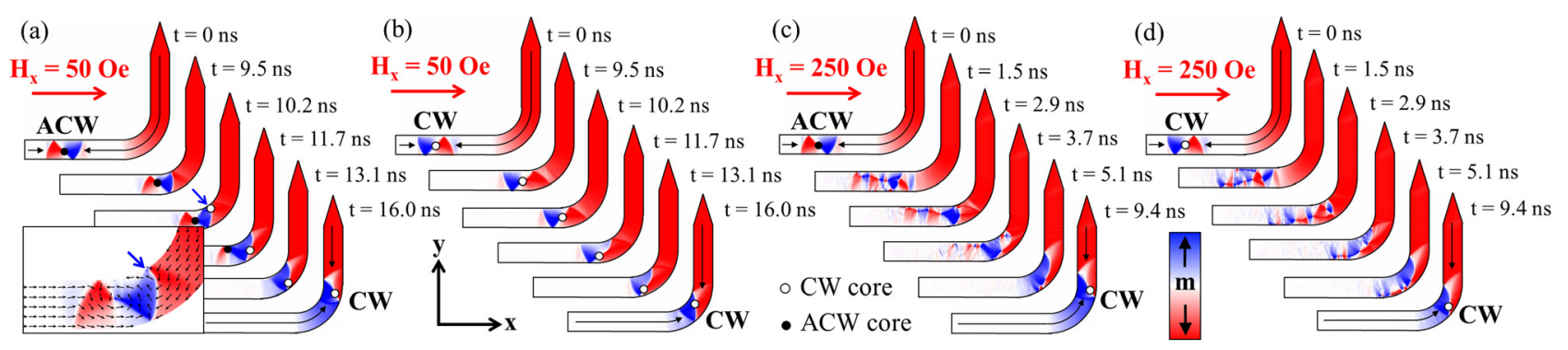

FIG. 2. Micromagnetic simulations of VDWs passing through corners with $\mathrm{r}=1 \mu \mathrm{m}$ in a $400 \mathrm{~nm}$ wide, $40 \mathrm{~nm}$ thick $\mathrm{Ni}_{80} \mathrm{Fe}_{20}$ nanowire. (a) $\mathrm{H}_{\mathrm{x}}=50 \mathrm{Oe}, \mathrm{ACW}$ initial VDW chirality. The inset figure shows an enlargement of the position at which the rectified vortex core nucleates (b) $\mathrm{H}_{\mathrm{x}}=50 \mathrm{Oe}$, CW initial VDW chirality. (c) $\mathrm{H}_{\mathrm{x}}=250 \mathrm{Oe}$, ACW initial VDW chirality. (d) $\mathrm{H}_{\mathrm{x}}=250 \mathrm{Oe}, \mathrm{CW}$ initial VDW chirality. The locations of the cores of CW and ACW vortices are identified with open and closed symbols, respectively, except in the intermediate images of (c) and (d) where the magnetization dynamics are highly turbulent.

nanowire was magnetized along $+\mathrm{y}$ rather than $-\mathrm{y}$ and would align with leading edge of a ACW VDW, causing rectification to that chirality. Following this logic, changing from $\mathrm{H} 2 \mathrm{H}$ to tail-to-tail (T2T) DWs should also switch the nature of the rectification, and this is demonstrated for an UP geometry nanowire in Fig. 1(f) where we show a T2T CW VDW being rectified to $\mathrm{ACW}$ chirality rather than maintaining its $\mathrm{CW}$ chirality as would have occurred for a H2H VDW.

To probe how the rectification process depended on the geometries of the corners and the applied field, we performed further simulations with a range of corner radii and propagation fields for $320 \mathrm{~nm}$ wide nanowires. For corners with $\mathrm{r}>2 \mu \mathrm{m}$, we used the graphics processing unit accelerated MUMAX ${ }^{3}$ software package ${ }^{19}$ to decrease the computation time. For each value of $r$ simulated, we found a critical field, $\mathrm{H}_{\mathrm{R}}$, below which the rectification process was suppressed. We note that this is a slightly counter-intuitive result: typically, the reliability of DW processes breaks down at high fields, where WB destabilizes DW structure. However, here such instability was critical to the nucleation of vortices at DW's leading edge (and thus rectification), hence the onset of the rectifying behavior above a critical field value. The values of $\mathrm{H}_{\mathrm{R}}$ were first established roughly by raising $\mathrm{H}_{\mathrm{x}}$ in $50 \mathrm{Oe}$ increments until reliable rectification was observed, before further simulations were performed to refine $H_{R}$ to within \pm 12.5 Oe. Data presenting $H_{R}$ as a function of $r$ is shown in Fig. 3(a). For $r \geq 1.6 \mu \mathrm{m}, H_{R}$ was poorly defined, with rectification occurring sporadically over a range of fields (marked with open symbols). In these geometries, we suggest the rectification process was sensitive to the magnetization configuration of the DW, in addition to the magnitude of the applied field. As the DWs were propagating above WB in all of the simulations presented, slight variations in their structures when encountering the corners would be expected. However, despite these complexities, the data still clearly showed $\mathrm{H}_{\mathrm{R}}$ to increase with $\mathrm{r}$.

To test the predictions of the simulations, we measured two further structures: an UP nanowire with $\mathrm{r}=2 \mu \mathrm{m}$ and $\mathrm{g}=160 \mathrm{~nm}$, and a DOWN nanowire with $\mathrm{r}=5 \mu \mathrm{m}$ and no notch. In the $\mathrm{r}=2 \mu \mathrm{m}$ device, all VDWs rectified to $\mathrm{CW}$ chirality, as would be expected given that $\mathrm{H}_{\mathrm{x}}=250-300 \mathrm{Oe}$ was required to depin the VDWs from the notch. In the $\mathrm{r}=5 \mu \mathrm{m}$ device, DWs propagated directly from the pad to the corner at $\mathrm{H}_{\mathrm{x}} \sim 180 \mathrm{Oe}$ which, considering the trend in Fig. 3(a), was close to the boundary between stochastic and deterministic rectification. In a total of 19 imaging cycles of this device, we observed one instance of a VDW with CW chirality being produced rather than the favored ACW chirality (Fig. 3(b)), adding evidence to validity of our simulations.

For applications where data are encoded using the chirality of VDWs, ${ }^{11}$ it would be necessary to propagate DWs around corners without chirality modification. Fig. 3(a) indicates this would only be possible with $\mathrm{H}_{\mathrm{x}}<50$ Oe unless the corners had high radii. However, the damping constant of

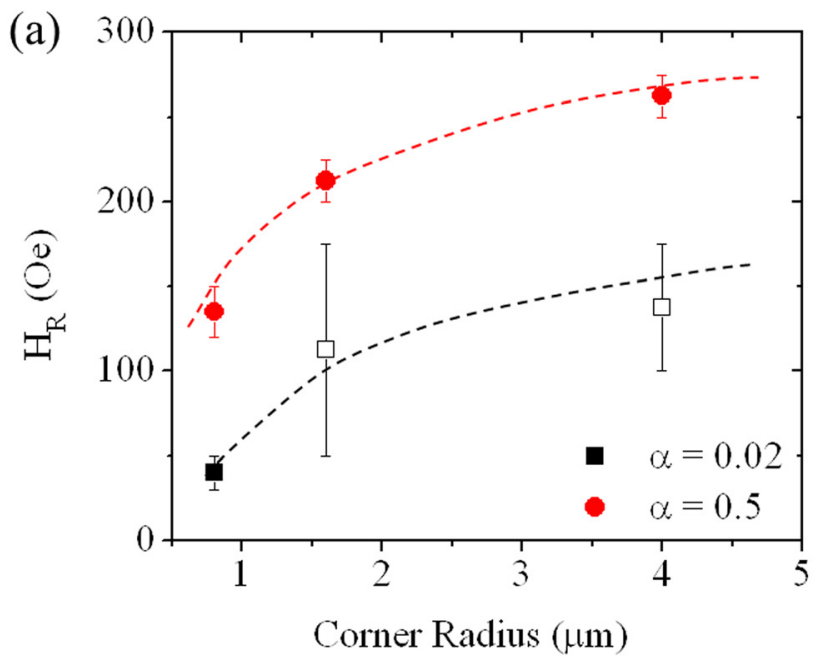

(b)

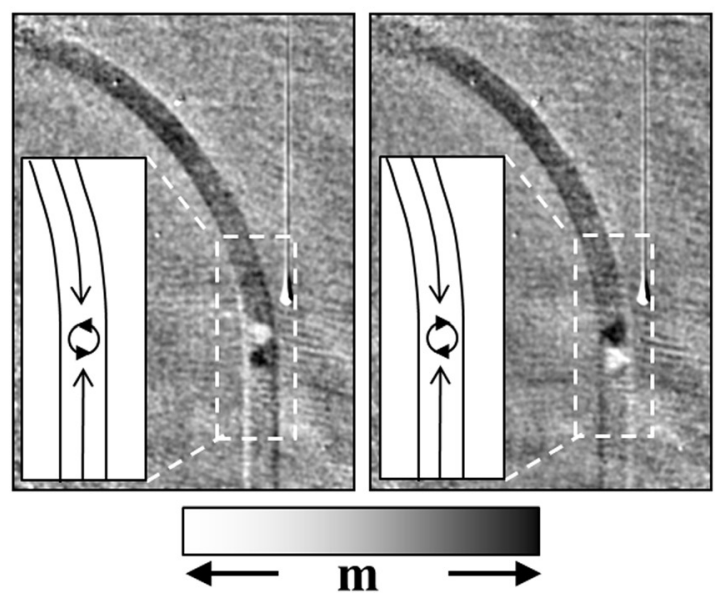

FIG. 3. (a) Plot showing the simulated field required to rectify VDWs $\left(\mathrm{H}_{\mathrm{R}}\right)$ as a function of corner radius for $320 \mathrm{~nm}$ wide, $40 \mathrm{~nm}$ thick $\mathrm{Ni}_{80} \mathrm{Fe}_{20}$ nanowires. Data are shown for damping constants $\alpha=0.02$ (squares) and $\alpha=0.5$ (circles). Where open symbols are used the error bars indicate a field range over which rectification was seen sporadically. (b) MTXM images showing ACW and CW VDW configurations formed after the corner of a "DOWN" geometry nanowire with $\mathrm{r}=5 \mu \mathrm{m}$ and no notch. 
$\mathrm{Ni}_{80} \mathrm{Fe}_{20}$ can be enhanced to $\alpha=0.3-0.8$ by doping with rare earth elements, ${ }^{20,21}$ causing DWs to propagate more rigidly ${ }^{22}$ and thus be less susceptible to chirality modification. Fig. 3(a) also presents data showing the variation of $\mathrm{H}_{R}$ with $r$ for $\alpha=0.5$. With enhanced damping $\mathrm{H}_{\mathrm{R}}>100$ Oe even for corners with $\mathrm{r}<1 \mu \mathrm{m}$. Thus, modifying damping provides a route to stabilizing chirality-encoded information in complex nanowire networks.

Having shown the ability of corners to rectify the chiralities of VDWs, we turned our attention to demonstrating their functionality in device designs. DWs pinned at artificial defect sites typically exhibit stochastic depinning field distributions, due to DWs pinning in a variety of configurations. These configurations can be DWs with different internal magnetization structures $^{23}$ (i.e., transverse DW (TDW) or vortex) or with different chiralities. ${ }^{8,9}$ Choosing nanowire geometries that strongly favor TDWs or VDWs should prevent structural variations; however, eliminating chirality dependent effects is more challenging, requiring both control of the initial DW chiralities and WB modes that conserve chirality.

Fig. 4(a) presents a simulation of an ACW VDW propagating under $\mathrm{H}_{\mathrm{x}}=50 \mathrm{Oe}$, performed using a $2.5 \mathrm{~nm} \times$ $2.5 \mathrm{~nm} \times 10 \mathrm{~nm}$ mesh. The dynamics consisted of periodic elongations and contractions of the DW, with multiple vortices and anti-vortices forming along its central boundary during the elongation phase. Critically, the basic symmetry of the DW was maintained at all times so that its chirality was always conserved on contracting back to VDW structure. Furthermore, the energy of a bi-domain state containing a TDW was $26 \%$ higher than one containing a VDW making the pinning of TDWs at defect sites highly unlikely. Therefore, it was expected that by combining this nanowire geometry with the rectifying effects of the corner we could suppress stochastic DW pinning.

To demonstrate this, we fabricated nanowires with the geometries labeled "STRAIGHT," "UP," and "DOWN" in Figs. 4(b)-4(d). Each nanowire contained a $60 \mathrm{~nm}$ deep triangular notch where the stochasticity of DW pinning could be probed. Based on our previous results, we expected that T2T VDWs in the "UP" devices would rectify to ACW chirality at the $\mathrm{r}=1 \mu \mathrm{m}$ corners, while those in the "DOWN" geometry would rectify to $\mathrm{CW}$ chirality. We characterized the nanowires' switching using a focused magneto-optic Kerr effect magnetometer. DW injection and depinning field distributions were measured by performing 100 single shot measurements at the locations indicated in Fig. 4.

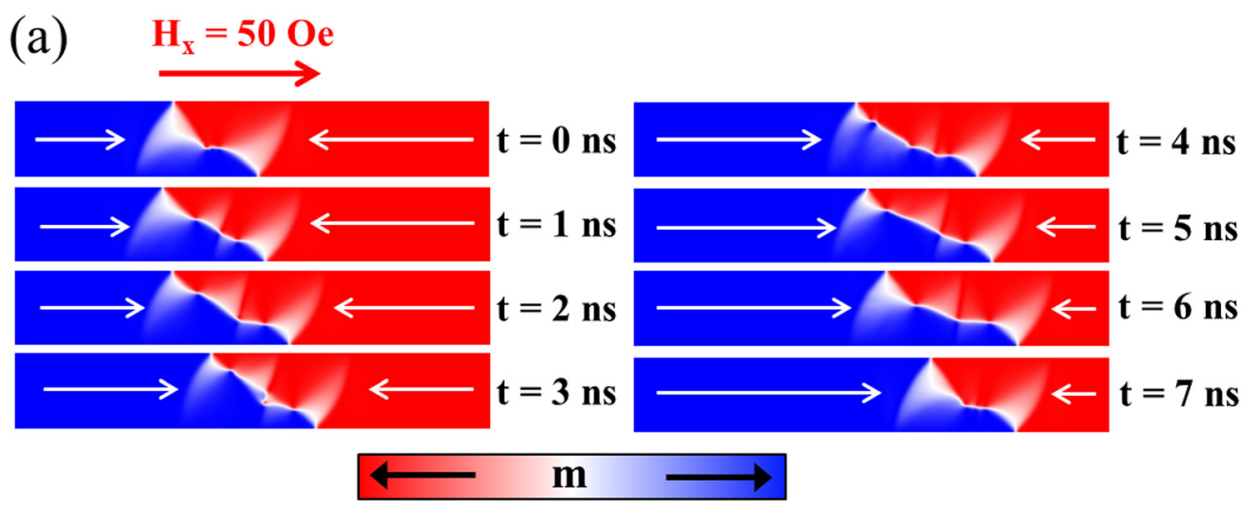

(b)

(c)

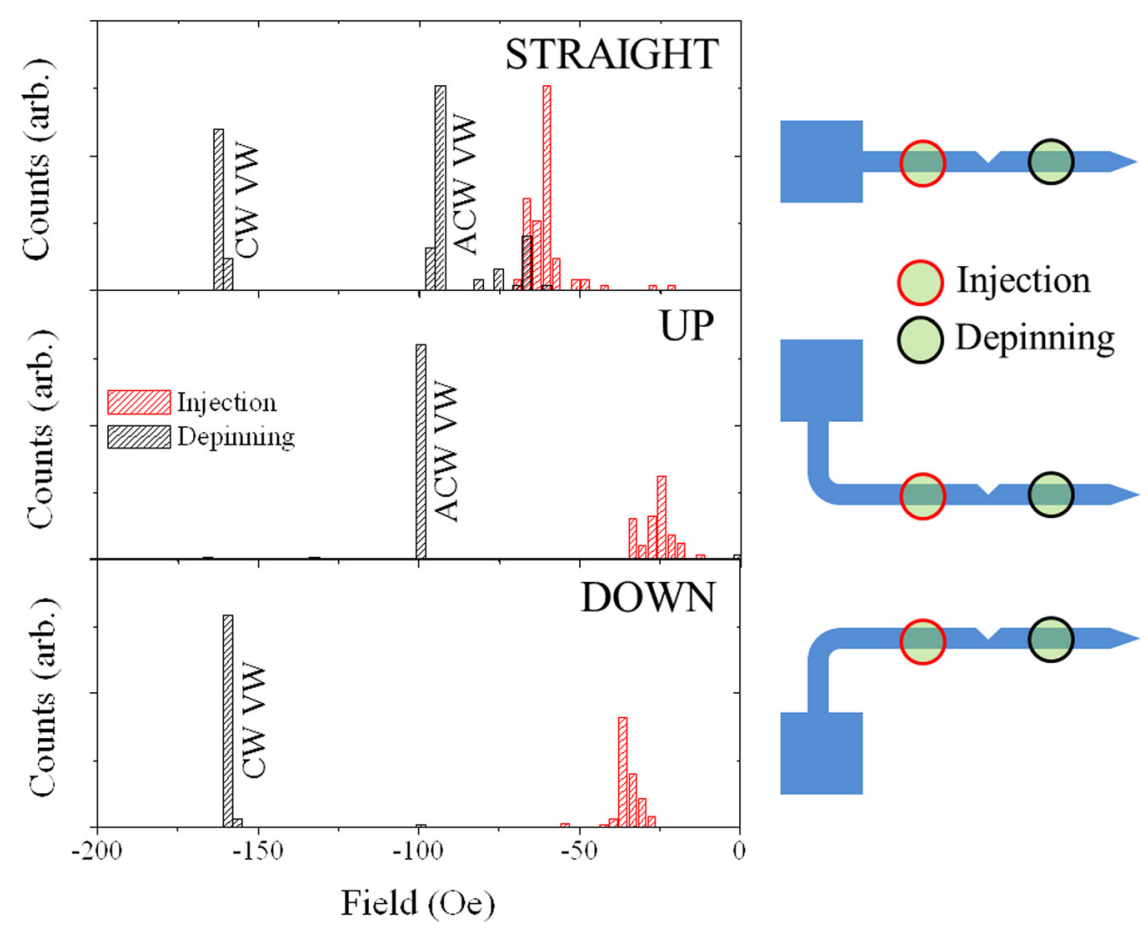

FIG. 4. (a) Simulated VDW dynamics at $\mathrm{H}_{\mathrm{x}}=50 \mathrm{Oe}$ in a $400 \mathrm{~nm}$ wide, $40 \mathrm{~nm}$ thick $\mathrm{Ni}_{80} \mathrm{Fe}_{20}$ nanowire. The images show a full period of the Walker breakdown oscillations. (b)-(d) Injection and depinning field distributions measured for (b) "STRAIGHT," (c) "UP," and (d) "DOWN" geometry nanowires. The schematic diagrams on the left of the figures indicate the positions of the laser spot for the injection and depinning field measurements. 
Fig. 4(b) shows data obtained from a "STRAIGHT" nanowire, where a mixture of $\mathrm{CW}$ and $\mathrm{ACW}$ DWs were expected to pin at the notch. The depinning field distribution showed a high degree of stochasticity with two clear peaks separated by $\sim 170$ Oe. Based on the results of micromagnetic simulations of VDW depinning fields, we assigned the peak at the lower field to ACW VDWs and the peak at the higher field to CW VDWs. Figs. 4(c) and 4(d) present the results of equivalent measurements on the "UP" and "DOWN" nanowires. Both nanowires exhibited depinning field distributions with a single peak, corresponding to the expected ACW VDWs for the "UP" geometry and CW VDWs for the "DOWN" geometry. We also performed similar measurements on two further devices of each geometry. Across three "STRAIGHT" nanowires, we observed a relatively even split between depinning events assigned to ACW and CW VDWs (45\% ACW to 55\% CW). For the "UP" nanowires, we observed a strong bias towards ACW VDWs (84\% ACW to $16 \% \mathrm{CW}$ ), while for the "DOWN" nanowires, we observed the opposite trend (94\% $\mathrm{CW}$ to $6 \% \mathrm{ACW})$. Cumulatively, these results indicate the feasibility of using nanowire corners to suppress stochastic pinning behavior.

In summary, we have shown that the chiralities of VDWs in magnetic nanowires are rectified when they are propagated ballistically into sharp nanowire corners, with the sense of rectification being determined by whether the nanowires turn left or right. In contrast to previously demonstrated methods of controlling the chirality of propagating DWs,${ }^{13-15}$ this approach can be implemented in continuous, defect-free, nanowires. Rectification can be suppressed by increasing the radii of nanowire corners, propagating VDWs at low fields, or by increasing the damping constant of the material from which the nanowires are patterned. We have also demonstrated how our approach can be combined with modes of DW propagation that conserve vortex chirality to dramatically reduce the stochasticity of DW pinning at artificial defect sites.

In addition to providing a tool to control VDW chirality in experimental studies this will be useful in chirality-based logic devices ${ }^{11}$ where the chiralities of VDWs must be switched as they propagate through complex nanowire networks. Furthermore, corners could be used to "reset" DW chirality to suppress stochastic pinning effects in more conventional devices where data is encoded using the orientation of magnetic domains.

This work was supported by the EPSRC (Grant No. EP/ J002275/1). The operation of the microscope was supported by the Director, Office of Science, Office of Basic Energy Sciences, Materials Sciences and Engineering Division, of the U.S. Department of Energy under Contract No. DEAC02-05-CH11231. P.F. and M.-Y.I. acknowledge support by the Leading Foreign Research Institute Recruitment Program (Grant No. 2012K1A4A3053565) through the National Research Foundation of Korea (NRF) funded by the Ministry of Education, Science and Technology (MEST).

${ }^{1}$ G. Hrkac, J. Dean, and D. A. Allwood, Philos. Trans. R. Soc., A 369, 3214 (2011).

${ }^{2}$ S. S. P. Parkin, M. Hayashi, and L. Thomas, Science 320, 190 (2008).

${ }^{3}$ D. A. Allwood, G. Xiong, C. C. Faulkner, D. Atkinson, D. Petit, and R. P. Cowburn, Science 309, 1688 (2005).

${ }^{4}$ R. D. McMichael and M. J. Donahue, IEEE Trans. Magn. 33, 4167 (1997).

${ }^{5}$ N. L. Schryer and L. R. Walker, J. Appl. Phys. 45, 5406 (1974).

${ }^{6}$ J.-Y. Lee, K.-S. Lee, S. Choi, K. Y. Guslienko, and S.-R. Kim, Phys. Rev. B 76, 184408 (2007).

${ }^{7}$ S.-K. Kim, J.-Y. Lee, Y.-S. Choi, K. Y. Guslienko, and K.-S. Lee, Appl. Phys. Lett. 93, 052503 (2008).

${ }^{8}$ M. Hayashi, L. Thomas, C. Rettner, R. Moriya, X. Jiang, and S. S. P. Parkin, Phys. Rev. Lett. 97, 207205 (2006).

${ }^{9}$ L. K. Bogart, D. Atkinson, K. O'Shea, D. McGrouther, and S. McVitie, Phys. Rev. B 79, 054414 (2009).

${ }^{10}$ A. Pushp, T. Phung, C. Rettner, B. P. Hughes, S.-H. Yang, L. Thomas, and S. S. P. Parkin, Nat. Phys. 9, 505 (2013).

${ }^{11}$ K. A. Omari and T. J. Hayward, Phys. Rev. Appl. 2, 044001 (2014).

${ }^{12}$ D. McGrouther, S. McVitie, J. N. Chapman, and A. Gentils, Appl. Phys. Lett. 91, 022506 (2007).

${ }^{13}$ E. R. Lewis, D. Petit, L. O'Brien, A. Fernandez-Pacheco, J. Sampaio, A. V. Jausovec, H. T. Zeng, D. E. Read, and R. P. Cowburn, Nat. Mater. 9, 980 (2010).

${ }^{14}$ J. Brandão, R. L. Novak, H. Lozano, P. R. Soledade, A. Mello, F. Garcia, and L. C. Sampaio, J. Appl. Phys. 116, 193902 (2014).

${ }^{15}$ E.-S. Wilhelm, D. McGrouther, L. Heyne, A. Bisig, and M. Kläui, Appl. Phys. Lett. 95, 252501 (2009).

${ }^{16}$ A. Bisig, M.-A. Mawass, M. Stärk, C. Moutafis, J. Rhensius, J. Heidler, S. Gliga, M. Weigand, T. Tyliszczak, B. Van Waeyenberge, H. Stoll, G. Schütz, and M. Kläui, Appl. Phys. Lett. 106, 122401 (2015).

${ }^{17}$ The 28 imaging cycles break down as follows: DOWN nanowires-8 imaging cycles, input ( $3 \mathrm{CW}, 3 \mathrm{ACW}, 2$ indeterminate), output $(0 \mathrm{CW}, 8$ $\mathrm{ACW})$. UP nanowires-20 imaging cycles, input (10 CW, $6 \mathrm{ACW}, 4$ indeterminate), output (20 CW, $0 \mathrm{ACW})$.

${ }^{18}$ OOMMF User's Guide, Version 1.0, Interagency Report NISTIR 6376, edited by M. J. Donahue and D. G. Porter (National Institute of Standards and Technology, Gaithersburg, MD, 1999).

${ }^{19}$ A. Vansteenkiste, J. Leliaert, M. Dvornik, F. Garcia-Sanchez, and B. Van Waeyenberge, AIP Adv. 4, 107133 (2014).

${ }^{20}$ G. Woltersdorf, M. Kiessling, G. Meyer, J. U. Thiele, and C. H. Back, Phys. Rev. Lett. 102, 257602 (2009).

${ }^{21}$ W. Bailey, P. Kabos, F. Mancoff, and S. Russek, IEEE Trans. Magn. 37, 1749 (2001).

${ }^{22}$ T. A. Moore, P. Mohrke, L. Heyne, A. Kaldun, M. Kläui, D. Backes, J. Rhensius, L. J. Heyderman, J. U. Thiele, G. Woltersdorf, A. F. Rodriguez, F. Nolting, T. O. Mentes, M. A. Nino, A. Locatelli, A. Potenza, H. Marchetto, S. Cavill, and S. S. Dhesi, Phys. Rev. B 82, 094445 (2010).

${ }^{23}$ M.-Y. Im, L. Bocklage, P. Fischer, and G. Meier, Phys. Rev. Lett. 102, 147204 (2009). 